PROCEEDINGS OF THE

AMERICAN MATHEMATICAL SOCIETY

Volume 126, Number 8, August 1998, Pages 2345-2349

S 0002-9939(98)04655-3

\title{
A LERAY-SCHAUDER TYPE THEOREM FOR APPROXIMABLE MAPS: A SIMPLE PROOF
}

\author{
H. BEN-EL-MECHAIEKH, S. CHEBBI, AND M. FLORENZANO \\ (Communicated by Palle E. T. Jorgensen)
}

\begin{abstract}
We present a simple and direct proof for a Leray-Schauder type alternative for a large class of condensing or compact set-valued maps containing convex as well as nonconvex maps.
\end{abstract}

The aim of this note is to extend the Leray-Schauder type nonlinear alternative presented in $[\mathrm{BI}]$ to a condensing upper semicontinuous approximable set-valued map $F: X \rightarrow E$ when $X$ is a closed subset with nonempty interior of a locally convex topological vector space $E$. The proof presented here is even shorter and simpler than the one given in $[\mathrm{BI}]$.

In what follows $E$ stands for a Hausdorff locally convex topological vector space with a fundamental basis $\mathcal{N}$ of convex, symmetric neighborhoods of the origin; if $X, Y$ are nonempty subsets of $E$, then $F: X \rightarrow Y$ is a set-valued map with nonempty values (simply called map). The boundary, the interior, the closure, and the convex hull of a subset $A$ in $E$ are denoted by $\partial A$, int $A, \bar{A}$, and $\operatorname{co} A$ respectively.

Definition 1. $F$ is said to be upper semicontinuous (u.s.c.) on $X$ if and only if for any open subset $V$ of $Y$, the set $\{x \in X: F(x) \subset V\}$ is open in $X$.

Definitions 2 ([BD], [BI], see also [GGK] for metric spaces). (1) Given $U, V \in \mathcal{N}$, a function $s: X \rightarrow Y$ is said to be a $(U, V)$-approximative selection of $F$ if for any $x \in X, s(x) \in(F[(x+U) \cap X]+V) \cap Y$.

(2) $F: X \rightarrow Y$ is said to be approachable if it has a continuous $(U, V)$ approximative selection for any $(U, V) \in \mathcal{N} \times \mathcal{N} \cdot \mathcal{A}(X, Y)$ denotes the class of such maps. We write $\mathcal{A}(X)$ for $\mathcal{A}(X, X)$.

(3) $F$ is said to be approximable if its restriction $\left.F\right|_{K}$ to any compact subset $K$ of $X$ is approachable.

Note that an approachable map is approximable (cf. [B]).

Examples. It is well-known that if $F$ is u.s.c. with nonempty convex values, then $F$ is approachable provided $X$ is paracompact and $Y$ is convex (cf. [DG]). Obviously, $F$ is approximable without conditions on $X$ (see $[\mathrm{C}]$ ).

Received by the editors August 12, 1996 and, in revised form, January 16, 1997.

1991 Mathematics Subject Classification. Primary 47H04, 47H10, 54C60.

Key words and phrases. Leray-Schauder alternative, approximable set-valued maps, condensing, compact.

(C)1998 American Mathematical Society 
In the absence of convexity, we have:

Assume that $X$ is dominated by finite polyhedra (e.g. $X$ is a compact $A N R$ ) and that $F: X \rightarrow Y$ is a u.s.c. map with nonempty compact values. Then $F$ is in $\mathcal{A}(X, Y)$ if either one of the following situations holds:

(a) $Y$ is an $A N R$ and the values of $F$ are contractible ([BD]).

(b) The values of $F$ are $\infty$-proximally connected in $Y$ ([GGK]), see also [BD]).

Definitions 3 ([PF], see also $[\mathrm{CF}])$. (1) If $C$ is a lattice with a minimal element, denoted by 0 , a function $\Phi: 2^{E} \rightarrow C$ is called a measure of noncompactness provided that the following conditions hold for any $A, B \in 2^{E}$ :

(i) $\Phi(\overline{\mathrm{co}}(A))=\Phi(A)$;

(ii) $\Phi(A)=0$ if and only if $A$ is precompact;

(iii) $\Phi(A \cup B)=\max \{\Phi(A), \Phi(B)\}$.

(2) $F: X \rightarrow Y$ is said to be $\Phi$-condensing provided that if $A \subset X$ with $\Phi(F(A)) \geq \Phi(A)$, then $A$ is relatively compact.

It should be noticed that there exist $\Phi$-condensing maps $F: X \rightarrow E$ only if, for the subsets of $X$, precompactness coincides with relative compactness. On the other hand, a compact map $F: X \rightarrow E$ is $\Phi$-condensing if either the domain $X$ is complete or if $E$ is quasicomplete. Every map defined on a compact set is necessarily $\Phi$-condensing.

The following finite-type approximation property of compact approximable maps plays a crucial role in the proof of the main theorem of this note.

Lemma 1. Let $F: X \rightarrow E$ be a compact approximable map. Given any $V \in \mathcal{N}$, there exist a finite subset $N_{V}$ of $\overline{F(X)}$ and an approximable map $F_{V}$ with values in $\operatorname{co}\left(N_{V}\right)$ such that $F_{V}(x) \subset F(x)+V$, for every $x \in X$. Moreover, $F_{V}$ is u.s.c. with nonempty closed values whenever $F$ has the same properties.

If $F \in \mathcal{A}(X, E)$ takes its values in a convex compact subset $K$ of $E$, then $F \in$ $\mathcal{A}(X, K)$.

Proof. Let $V \in \mathcal{N}$ be arbitrary, and let $N_{V}=\left\{y_{1}, \ldots, y_{n}\right\}$ be a finite subset of $\overline{F(X)}$ such that the collection $\left\{y_{i}+\frac{1}{6} V: i=1, \ldots, n\right\}$ forms an open cover of the compact set $\overline{F(X)}$. Consider the Schauder projection (cf. [DG]) $\pi_{V}: \bigcup_{i=1}^{n}\left(y_{i}+\frac{1}{3} V\right) \rightarrow$ $\operatorname{co}\left(N_{V}\right)$ defined by:

$$
\pi_{V}(y):=\frac{1}{\sum_{i=1}^{n} \mu_{i}(y)} \sum_{i=1}^{n} \mu_{i}(y) y_{i}, \quad \text { for all } y \in \bigcup_{i=1}^{n}\left(y_{i}+\frac{1}{3} V\right),
$$

where $\mu_{i}(y)=\max \left\{0,1-p_{\frac{1}{3} V}\left(y-y_{i}\right)\right\}$ and $p_{\frac{1}{3} V}$ is the Minkowski functional of $\frac{1}{3} V$. One readily verifies that:

$$
\pi_{V}(y)-y \in \frac{1}{3} V, \quad \text { for all } y \in \bigcup_{i=1}^{n}\left(y_{i}+\frac{1}{3} V\right) .
$$

Define the map $F_{V}: X \rightarrow \operatorname{co}\left(N_{V}\right)$ as the composition product $F_{V}:=\pi_{V} \circ F$. If $F$ is approximable, $F_{V}$ is approximable since its restriction to any compact subset of $X$ is approachable as the composition product of two approachable maps (see [B, Proposition 2.5]). Moreover, $F_{V}(x) \subset F(x)+V$ for all $x \in X$, and $F_{V}$ is u.s.c. and it has nonempty compact values whenever $F$ has the same properties.

If $F \in \mathcal{A}(X, E)$ has values in a convex compact subset $K$ of $E$, for a given $U \in \mathcal{N}$, let $s: \quad X \rightarrow E$ be a continuous $\left(U, \frac{1}{6} V\right)$-approximative selection of $F$. Then one 
has for all $x \in X$,

$$
\begin{gathered}
s(x) \in F((x+U) \cap X)+\frac{1}{6} V \subset \bigcup_{i=1}^{n}\left(y_{i}+\frac{1}{6} V\right)+\frac{1}{6} V=\bigcup_{i=1}^{n}\left(y_{i}+\frac{1}{3} V\right), \\
\pi_{V}(s(x)) \in s(x)+\frac{1}{3} V \subset F((x+U) \cap X)+\frac{1}{6} V+\frac{1}{3} V \subset F((x+U) \cap X)+V .
\end{gathered}
$$

That is, $\pi_{V} \circ s$ is a continuous $(U, V)$-approximative selection of $F$ with values in $\operatorname{co}\left(N_{V}\right) \subset K$.

We shall also need the following generalization of the Fan-Kakutani fixed point theorem.

Lemma 2 ([BD]). Assume that $X$ is convex and compact in $E$ and that $F \in \mathcal{A}(X)$ is u.s.c. with nonempty closed values. Then $F$ has a fixed point, that is, a point $x_{0} \in X$ with $x_{0} \in F\left(x_{0}\right)$.

Finally, we prove for condensing maps the following useful result (see $[\mathrm{PF}]$ ).

Lemma 3. Assume that $X$ is a nonempty subset of $E$ and that $F: X \rightarrow E$ is a $\Phi$-condensing map. Then there exists a nonempty compact and convex subset $K$ of $E$ such that $F(K \cap X) \subset K$.

Proof. Let $x_{0} \in X$ be fixed. Let us consider the family $\mathcal{F}$ of all closed convex subsets $C$ of $E$ such that $x_{0} \in C$ and $F(C \cap X) \subset C$. Clearly $\mathcal{F} \neq \varnothing$, since $\overline{\mathrm{co}}\left(F(X) \cup\left\{x_{0}\right\}\right) \in \mathcal{F}$. Let $K=\bigcap_{C \in \mathcal{F}} C$. $K$ is convex and closed and $x_{0} \in K$. If $x \in K \cap X, F(x) \subset C$ for all $C \in \mathcal{F}$, so that $F(K \cap X) \subset K$ and thus $K \in \mathcal{F}$. It remains to prove that $K$ is compact. If $K$ is not compact, then $\Phi(F(K)) \nsupseteq \Phi(K)$, since $F$ is $\Phi$-condensing. Let $K^{\prime}=\overline{\operatorname{co}}\left(\left\{x_{0}\right\} \cup F(K \cap X)\right)$. Then $K^{\prime} \subset K$ which implies that $F\left(K^{\prime} \cap X\right) \subset F(K \cap X) \subset K^{\prime}$. Hence $K^{\prime} \in \mathcal{F}$ and $K \subset K^{\prime}$. Therefore $K=K^{\prime}, \Phi(K)=\Phi\left(K^{\prime}\right)=\Phi(F(K \cap X)) \leq \Phi(F(K))$ which contradicts $\Phi(F(K)) \nsupseteq \Phi(K)$.

We are ready now to present the main result of this note.

Theorem. Assume that $X$ is a closed subset of $E$ with boundary $\partial X$ and that 0 is an interior point of $X$. Let $F: X \rightarrow E$ be a $\Phi$-condensing or compact u.s.c. approximable map with nonempty closed values. Then one of the following properties holds:

(1) $\exists x_{0} \in X$, with $x_{0} \in F\left(x_{0}\right)$;

(2) $\exists \hat{x} \in \partial X, \exists \lambda \in(0,1)$, with $\hat{x} \in \lambda F(\hat{x})$.

Proof. Case 1. $F$ is $\Phi$-condensing.

Suppose for each $x \in X, x \notin F(x)$ and for each $(\lambda, x) \in(0,1) \times \partial X, x \notin \lambda F(x)$. By Lemma 3, there exists a nonempty convex and compact subset $K$ of $E$ such that $F(K \cap X) \subset K$. Without loss of generality we can assume that $0 \in K$. Since $K \cap X$ is compact, $\left.F\right|_{K \cap X} \in \mathcal{A}(K \cap X, E)$ and, by Lemma $1,\left.F\right|_{K \cap X} \in \mathcal{A}(K \cap X, K)$. Let $F^{\prime}: K \rightarrow K$ be the map defined by:

$$
F^{\prime}(x):= \begin{cases}F(x) & \text { if } x \in \operatorname{int} X \\ K & \text { if } x \notin \operatorname{int} X\end{cases}
$$


$F^{\prime}$ is u.s.c. with nonempty closed values. We first claim that $F^{\prime} \in \mathcal{A}(K)$. Indeed, let $(U, V) \in \mathcal{N} \times \mathcal{N}$ be arbitrary and $s: K \cap X \rightarrow K$ be a continuous $\left(U, \frac{1}{2} V\right)$ approximative selection of $\left.F\right|_{K \cap X}$. By Proposition 1.6 of [BD], there exists a continuous function $s^{\prime}: K \rightarrow K$ such that $s$ and $\left.s^{\prime}\right|_{K \cap X}$ are $\frac{1}{2} V$-near. Therefore $s^{\prime}$ is a continuous $(U, V)$-approximative selection of $F^{\prime}$.

Consider now the set $C=\{x \in X \cap K \mid x \in \lambda F(x)$ for some $0 \leq \lambda \leq 1\}$. C is nonempty $(0 \in C)$ and $C$ is closed $(F$ is u.s.c. and $F(X \cap K) \subset K)$, hence compact. Since $E$ is Hausdorff, it is in fact a uniformizable space, hence completely regular (see $[\mathrm{K}]$, p. 47). Since $C \cap(E \backslash \operatorname{int} X)=\varnothing$, there is a continuous function $a: E \rightarrow[0,1]$ such that $a(x)=1$ for $x \in C$ and $a(x)=0$ for $x \in E \backslash \operatorname{int} X$. Let $G: K \rightarrow K$ be the map defined by:

$$
G(x):=a(x) F^{\prime}(x) .
$$

$G$ is u.s.c. with nonempty closed values and by Proposition 2.4 and Proposition 2.5 of [B], $G \in \mathcal{A}(K)$. By Lemma $2, G$ has a fixed point $x_{0} \in K, x_{0} \in a\left(x_{0}\right) F^{\prime}\left(x_{0}\right)$. If $x_{0} \notin \operatorname{int} X, a\left(x_{0}\right)=0$ and $x_{0}=0$, which contradicts the hypothesis $0 \in \operatorname{int} X$. If $x_{0} \in \operatorname{int} X, x_{0} \in a\left(x_{0}\right) F\left(x_{0}\right)$, hence $x_{0} \in C, a\left(x_{0}\right)=1$ and $x_{0}$ is a fixed point of $F$, another contradiction.

Case 2. $F$ is compact.

Let $V \in \mathcal{N}$ be arbitrary but fixed. Consider the finite subset $N_{V}$ of $\overline{F(X)}$ and the approximable map $F_{V}: X \rightarrow \operatorname{co}\left(N_{V}\right)$ verifying $F_{V}(x) \subset F(x)+V$, for all $x \in X$, both provided by Lemma 1 . Without loss of generality, we can assume that $0 \in \operatorname{co}\left(N_{V}\right)$ (otherwise, replace $\operatorname{co}\left(N_{V}\right)$ by $\left.\operatorname{co}\left(0 \cup N_{V}\right)\right)$ and note that $\left.F_{V}\right|_{X \cap \operatorname{co}\left(N_{V}\right)} \in$ $\mathcal{A}\left(X \cap \operatorname{co}\left(N_{V}\right), \operatorname{co}\left(N_{V}\right)\right)$.

The same proof as in Case 1 applied to $\left.F_{V}\right|_{X \cap \operatorname{co}\left(N_{V}\right)}$ in place of $\left.F\right|_{X \cap K}$ leads to the following alternative:

(1) $)_{V} \exists x_{V} \in X$, with $x_{V} \in F_{V}\left(x_{V}\right)$; or

$(2)_{V} \exists x_{V} \in \partial X, \exists \lambda_{V} \in(0,1)$ with $x_{V} \in \lambda_{V} F_{V}\left(x_{V}\right)$.

A straightforward argument (see [BI]) based on the compactness of $F$, its upper semicontinuity and the closedness of its values ends the proof.

We conclude the note with some remarks.

Remarks. (i) Obviously, if $X$ is complete or $E$ is quasicomplete, the result in the compact case follows from the result in the condensing case.

(ii) The previous result extends the Theorem in [R3] proved for convex-valued maps. Our proof is an adaptation of its proof. For $\Phi$-condensing convex-valued maps, the result was obtained in $[\mathrm{PF}]$ using a topological degree argument.

(iii) Note that for $F$ compact, the preceding theorem reduces to the Theorem in [BI]. However, the proof provided here is much shorter and simpler.

(iv) The single-valued condensing case was treated in [R1], [R2]. We also refer to $[\mathrm{DG}]$ for a treatment of the single valued compact case based on the theory of transversality and to $[\mathrm{BI}]$ for other references and comments.

(v) After acceptance of this note, reference $[\mathrm{P}]$ came to the authors' attention. There, Case 2 of our main theorem is treated, independently, with a similar argument.

\section{ACKNOWLEDGMENT}

The first author acknowledges the support of the Natural Sciences and Engineering Research Council of Canada. 


\section{REFERENCES}

[B] H. Bel El Mechaiekh, Continuous approximations of multifunctions, fixed points and coincidences, in Approximation and Optimization in the Carribean II, Proceedings of the Second International Conference on Approximation and Optimization in the Carribean, Florenzano et al. Eds., Verlag Peter Lang, Frankfurt, (1995), 69-97.

[BD] H. Ben-El-Mechaiekh and P. Deguire, Approachability and fixed points for non-convex set-valued maps, J. Math. Anal. Appl. 170 (1992), 477-500. MR 94a:54103

[BI] H. Bel-El-Mechaiekh and A. Idzik, A Leray-Schauder type theorem for approximable maps, Proc. Amer. Math. Soc. 122 (1994), 105-109. MR 94k:54074

[C] A. Cellina, A theorem on the approximation of compact multivalued mappings, Atti Accad. Naz. Lincei (8) 47 (1969), 429-433. MR 43:2676

[CF] S. Chebbi and M. Florenzano, Maximal elements and equilibria for condensing correspondences, Cahiers Eco-Math 95.18 (1995), CERMSEM, Université de Paris I, to appear in Nonlinear Analysis.

[DG] J. Dugundji and A. Granas, Fixed point theory I, Monografie Math. 61, PWN, Warszawa, 1982. MR 83j:54038

[GGK] L. Górniewicz, A. Granas and W. Kryszewski, Sur la methode de l'homotopie dans la theorie des points fixes pour les applications multivoques, Partie I: Transversalité topologique, C. R. Acad. Sci. Paris Sér. I Math. 307 (1988), 489-492; Partie II: L'indice dans les ANRs compacts, 308 (1989), 449-452. MR 90g:55002; MR 90j:55004

[K] G. Köthe, Topological Vector Spaces I, Springer-Verlag, New York Inc., 1969. MR 40:1750

[P] S. Park, Fixed points of approximable maps, Proc. Amer. Math. Soc. 124 (1996), 31093114. MR 96m: 47108

[PF] W. V. Petryshyn and P. M. Fitzpatrick, A degree theory, fixed point theorems and mapping theorems for multivalued noncompact mappings, Trans. Amer. Math. Soc. 194 (1974), 125.

[R1] S. Reich, Fixed points in locally convex spaces, Mat. Z. 125 (1972), 17-31. MR 46:6110

[R2] S. Reich, Fixed points of condensing functions, J. Math. Anal. Appl. 41 (1973), 460-467. MR 48:971

[R3] S. Reich, A remark on set-valued mapppings that satisfy the Leray-Schauder condition I, II, Atti Accad. Naz. Lincei 61 (1976), 193-194; 66 (1979), 1-2.

Department of Mathematics, Brock University, St. Catharines, Ontario, Canada L2S $3 \mathrm{~A} 1$

Current address: Department of Mathematics, The American University of Sharjah, P.O. Box 26666, Sharjah, United Arab Emirates

E-mail address: hmechaiekh@aus.ac.ae

CERMSem, Université de Paris I, 106-112 Bd de L'Hopital, 75013 Paris, France

E-mail address: chebbi@univ-parisl.fr

CNRS-CEPREMAP, 140 Rue du Chevaleret, 75013 Paris, France

E-mail address: monique.florenzano@cepremap.cnrs.fr 